\title{
陕西延安两个坡度不同土壤剖面磁学性质及其 在古降雨量重建中的意义
}

\author{
刘志锋 (1)2 ${ }^{(2)}$ 刘青松 ${ }^{(1) *}$ \\ (1) 中国科学院地质与地球物理研究所岩石圈演化国家重点实验室, 北京 100029; \\ (2) 中国科学院大学地球科学学院, 北京 100049 \\ * 联系人, E-mail: liux0272@yahoo.com
}

收稿日期: 2012-11-01; 接受日期: 2013-05-25; 网络版发表日期: 2013-11-22

国家自然科学基金项目(批准号: 40974036, 41025013)、国家自然科学基金创新研究群体项目(批准号: 40821091)、中国科学院创新团队国 际合作伙伴计划和中国科学院百人计划项目资助

\begin{abstract}
摘要 土壤具有空间分布广泛、时间分布连续的特点, 并且其物理化学性质与气候因素密 切相关，因此，可用于古气候重建. 以陕西延安两个距离相近、坡度不同的土壤剖面 LP 和 LPM(LP 水平, LPM 坡度为 $20^{\circ}$ ) 为研究对象, 利用环境磁学方法研究土壤剖面地形(地表坡度) 对其磁学性质的影响及对古气候重建的意义. 结果表明, LP 磁化率平均值和最大值均为 LPM 两倍, 两个剖面磁性矿物类型和粒径分布相似. 因此, 在应用传统磁学参数磁化率进行古降 雨量重建时, 同一地点的不同土壤剖面磁化率可能存在很大差异. 另外, LP与 LPM 两剖面因 成土作用引起的磁化率增强值与母质的比值分别为 2.27 和 2.04 , 饱和等温剩磁增强比值分别 为 1.80 和 1.86 , 两剖面比值相差不大. 将 LP 和 LPM 两个剖面频率磁化率 $\left(\chi_{\mathrm{FD}}\right)$ 及非磁滞剩磁 $(\mathrm{ARM})$ 和 “硬”等温剩磁强度 (HIRM) 进行线性拟合, 得到的线性拟合斜率 $\chi_{\mathrm{FD}} / \mathrm{HIRM}$ 和 ARM/HIRM, 包含成土作用生成的亚铁和反铁磁性矿物信息且受母质影响很小, LP 和 LPM 的 $\chi_{\mathrm{FD}} / \mathrm{HIRM}$ 拟合斜率分别为 $28.7 \times 10^{-5}$ 和 $28.9 \times 10^{-5} \mathrm{~A} \mathrm{~m}^{-1}, \mathrm{ARM} / \mathrm{HIRM}$ 分别为 0.35 和 0.33 . 这些 结果表明土壤剖面磁化率(或饱和等温剩磁)增强值、 $\chi_{\mathrm{FD}} / \mathrm{HIRM}$ 和 $\mathrm{ARM} / \mathrm{HIRM}$ 受土壤母质和 地形影响较小, 在大范围内用来重建古降雨量可能比传统磁学参数(如磁化率)更适合.
\end{abstract}

关键词

土壤剖面

坡度

磁学性质

古降雨量重建
土壤在空间分布广泛, 时间分布连续(黄土-古土 壤序列), 其物理化学性质与气候因素密切相关, 因 此, 可以被用来重建古气候变化历史(Evans 和 Heller, 2003; Maher，2007). 土壤剖面指从地表到母质的垂 直断面, 不同类型土壤, 其发生层、颜色、质地、结 构和粒度等特征差别很大(Chesworth, 2008). 根据土
壤发生学理论, 在土壤形成过程中, 其性质主要受地 形、母质、时间、生物和气候五个因素作用(Jenny, 1941)，在这几个成土因素的影响下，母岩或沉积物 发生各种物理化学变化, 导致其中各种矿物发生迁 移和转化, 分化成一系列组成、性质和形态各不相同 的层次, 称为发生层. 一般而言, 土壤剖面分为表土

中文引用格式: 刘志锋, 刘青松. 陕西延安两个坡度不同土壤剖面磁学性质及其在古降雨量重建中的意义. 中国科学: 地球科学, 2013, 43: 2037-2048 英文引用格式: Liu Z F, Liu Q S. Magnetic properties of two soil profiles from Yan'an, Shaanxi Province and their implications for paleorainfall reconstruction. Science China: Earth Sciences, 2013, doi: 10.1007/s11430-013-4781-4 
层( $\mathrm{A}$ 层)、淀积层 $(\mathrm{B}$ 层)和母质层 $(\mathrm{C}$ 层) 三个发生层. 研究表明, 成土过程中, 土壤中磁性矿物的种类 和含量随着气候因素而发生变化. 因此, 通过研究气 候因素对土壤磁学性质影响, 可以建立磁学性质和 气候之间的转换方程, 并进而应用土壤磁学参数定 量(半定量化)地研究古气候变化(Liu 等, 2012). 中国 西部的黄土高原是研究土壤磁学性质与古气候关系 的理想地点. Heller 等(1982)研究发现黄土与古土壤 序列的磁化率 $(\chi$, 质量归一化)与深海氧同位素有非 常好的对应性, $\chi$ 可以指示亚洲夏季风强度和降雨量 (Heller 和 Liu, 1984, 1986; Kukla 等, 1988; 刘秀铭等, 2007). Maher 等(1994)建立了 $\chi$ 与古降雨量之间的气 候转换函数, 通过使用成土磁化率 $\chi_{\text {ped }}$ (土壤剖面 $\mathrm{B}$ 层 与 $\mathrm{C}$ 层 $\chi$ 差值, 即 $\chi_{\mathrm{ped}}=\chi_{\mathrm{B}}-\chi_{\mathrm{C}}$ )减弱母质影响. Han 等 (1996)通过研究中国西南 63 个表层土壤 $\chi$, 发现 $\chi$ 不 仅与降雨量 (小于 $1100 \mathrm{~mm}$ )相关, 还可能受控于温度. 进一步研究发现, 在俄罗斯(Maher 等, 2002)和美国 (Geiss 等, 2008), 乃至在全球范围土壤 $\chi$ 都可以用来 指示降雨量(Balsam 等, 2011).

前人研究常用 $\chi$ 来重建古降雨量, 但是 $\chi$ 受母质 影响很大(Blundell 等, 2009), 限制了土壤磁学参数在 大范围重建古降雨量中的应用. 同时, 使用表层土壤 代替整个土壤剖面也可能造成误差. 另外, 研究表明 土壤所在的地形与地貌(比如地面坡度)对土壤磁学 特征也有显著影响, 因为坡度决定土壤剖面赋存的 水分含量和时间, 进而影响其风化的速率(Dixon 等, 2012). 为了进一步研究 $\chi$ 作为降雨量替代指标的可 信度, 本文选取了黄土高原同一地区(距离相近 100 $\mathrm{m}$ 左右)气候相同, 但是地面坡度不同两个土壤剖面 LP 和 LPM. 通过对比这两个剖面的磁学性质, 为应 用土壤磁学方法重建古降雨量提供参考.

\section{1 研究区域简介}

采样区位于陕西省延安市北部安塞县 $\left(36^{\circ} 35.4^{\prime} \mathrm{N}\right.$, $\left.109^{\circ} 9.1^{\prime} \mathrm{E}\right)$, 海拔 $1210 \mathrm{~m}$, 地处西北内陆黄土高原腹地, 鄂尔多斯盆地边缘, 属于典型的黄土高原丘陵沟壑区. 年平均温度 $8.9^{\circ} \mathrm{C}$, 降雨量 $510 \mathrm{~mm}$, 温带大陆性半干 旱季风气候. 7 月平均温度最高为 $23.4^{\circ} \mathrm{C}, 1$ 月平均温 度最低为零下 $5.5^{\circ} \mathrm{C} ; 70 \%$ 的降雨量分布在 $6 \sim 9$ 月, 8 月 的平均降雨量最高为 $118 \mathrm{~mm}, 1$ 月份平均降雨量最低 仅为 $3 \mathrm{~mm}$. 陕西省年均温度、降雨量如图 1(a)所示.

\section{2 样品采集与实验}

剖面 LP 发生学名称为黑垆土, 黑垆土是在黄土 高原半湿润-半干旱气候、黄土母质上、草原植被下 形成的土壤, 具有深厚的暗色腐殖质层, 有隐粘化特 征，全剖面有石灰反应，属钙层土纲(郭兆员，1992). LP 深度为 $202 \mathrm{~cm}$, 所处位置地表水平. 剖面 LPM 发 生学名称为黄绵土, 黄绵土是在黄土母质上直接形 成的幼年土壤, 土壤发育微弱, 剖面分异不明显, 其 形态和属性与黄土母质相近, 属初育土纲(郭兆员, 1992). LPM 深度为 $120 \mathrm{~cm}$, 地面坡度为 $20^{\circ}$ 左右. LP 和 LPM 剖面详细描述见表 1 , 采样间隔均为 $2 \mathrm{~cm}$, 母 质均为风尘沉积物. 样品采集好后装入样品袋运回 实验室, 在 $40^{\circ} \mathrm{C}$ 烘箱中干燥, 装入体积为 $8 \mathrm{~cm}^{3}$ 正方 体样品盒中, 固定好后进行磁学测量.

$\chi$ 使用捷克 Agico 公司生产的 MFK1-FA 多频磁 化率仪测量, 精度为 $2 \times 10^{-8} \mathrm{SI}$, 测量频率分别为 976, 3904 和 $15616 \mathrm{~Hz}$, 测量磁场 $200 \mathrm{Am}^{-1}$. 频率磁化率 $\left(\chi_{\mathrm{FD}}\right)$ 定义为 $\chi_{\mathrm{FD}}=\chi_{\mathrm{LF}}-\chi_{\mathrm{HF}}\left(\chi_{\mathrm{LF}}\right.$ 和 $\chi_{\mathrm{HF}}$ 分别为仪器在频率 976 和 $15616 \mathrm{~Hz}$ 测量得到的磁化率), 可以用来指示 样品中超顺磁 (SP) 与单畴 (SD) 边界附近的粘滞 SP(VSP)磁性颗粒含量(Dearing 等, 1996; 刘青松等, 2007). 频率磁化率百分比 $\chi_{\mathrm{FD}} \%$ (定义为 $\left(\chi_{\mathrm{LF}}-\chi_{\mathrm{HF}}\right) /$ $\left.\chi_{\mathrm{LF}} \times 100 \%\right)$ 常与 $\mathrm{SP}+\mathrm{SD}$ 颗粒的粒径分布有关(Worm, 1998; 刘青松和邓成龙, 2009). 非磁滞剩磁(ARM)对 样品中 SD 颗粒磁性矿物敏感(Dunlop 和 Özdemir, 1997), 使用 Enterprise 公司 $2 \mathrm{G}$ Enterprises model $760 \mathrm{R}$ 超导磁力仪测量, 交变退磁场为 $100 \mathrm{mT}$, 外加 直流场为 $0.05 \mathrm{mT}$.

等温剩磁(IRM)曲线和磁滞回线使用 Princeton 公司的振动磁力仪(VSM MicroMag TM 3900)测量. IRM 曲线测量时使用对数坐标, 初始磁场 $5 \mathrm{mT}$, 最 大外加磁场为 $1 \mathrm{~T}$, 共测量 100 个数据. 本文将样品 在 $1 \mathrm{~T}$ 磁场下获得的剩磁作为饱和等温剩余磁化强度 $(\mathrm{SIRM})$ ．然后，测量反向退磁曲线，得到反向场为 $0.3 \mathrm{~T}$ 时的剩磁强度和剩磁矫顽力 $\left(B_{\mathrm{cr}}\right)$. 磁滞回线测 量最大磁场为 $1 \mathrm{~T}$, 测量间隔为 $5 \mathrm{mT}$, 平均时间为 $200 \mathrm{~ms}$, 测量后得到磁滞回线参数矫顽力 $\left(B_{\mathrm{c}}\right)$ 、饱和 磁化强度 $\left(M_{\mathrm{s}}\right)$ 和剩余磁化强度 $\left(M_{\mathrm{rs}}\right)$. 计算 HIRM 和 S-ratio(软磁比) $\left(\mathrm{HIRM}=0.5 \times\left(\mathrm{SIRM}_{\mathrm{IR}} \mathrm{IR}_{-0.3 \mathrm{~T}}\right), \mathrm{S}\right.$-ratio= - IRM $\left._{-0.3 \mathrm{~T}} / \mathrm{SIRM}\right)$. HIRM 和 S-ratio 可以用来指示样 品中“硬”磁性矿物含量和“软”磁性矿物相对含量 


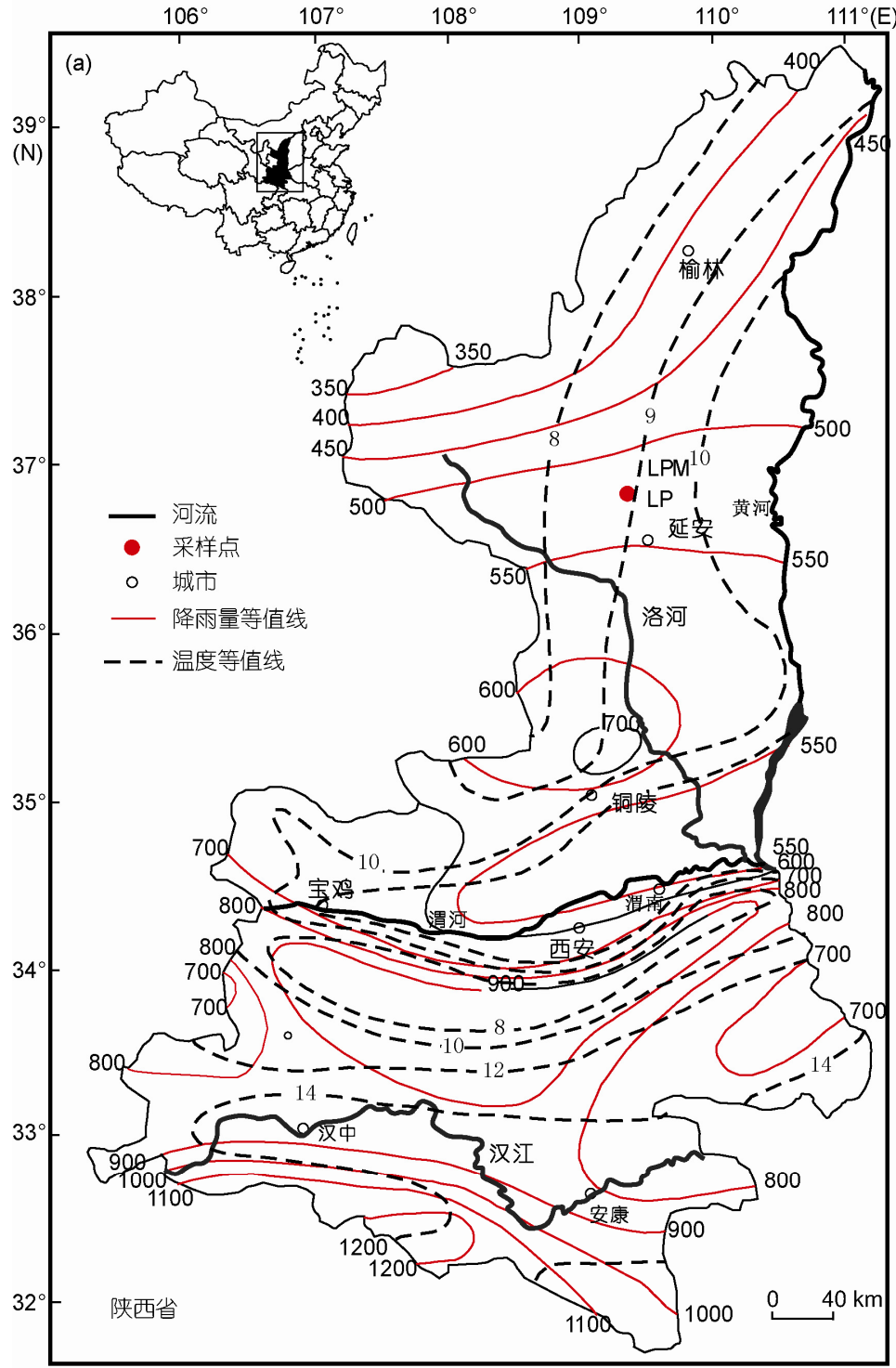

(b)

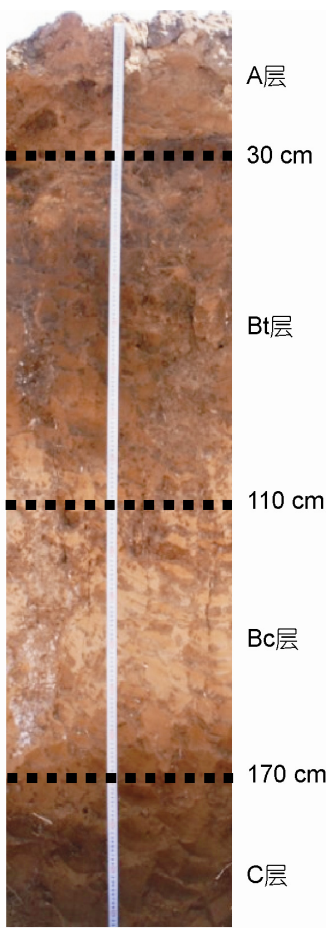

(c)

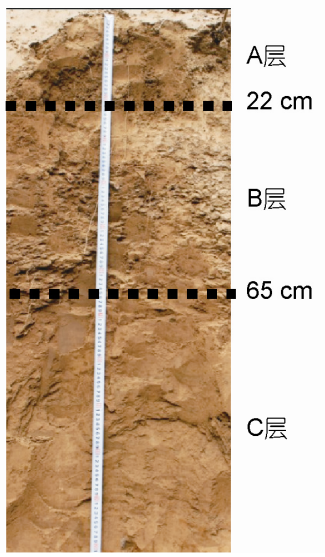

图 1 采样点地理位置及土壤剖面(a)、LP 剖面发生分层图(b)和 LPM 剖面发生分层图(c)

(a) 改自《陕西土壤》 (郭兆员, 1992); 图(b)和(c)中虚线为发生层分界线, 各发生层含义见表 1

表 1 LP 和 LPM 土壤剖面详细描述

\begin{tabular}{|c|c|c|c|c|c|c|}
\hline 剖面 & 深度(cm) & 发生层 & 颜色 & 质地 & 松紧度 & 根系 \\
\hline \multirow{4}{*}{ LP } & $0 \sim 31$ & 表层 & 7.5YR 6/6 & 轻壤 & 疏松 & 中 \\
\hline & $31 \sim 110$ & 粘化层 & 7.5YR 3/2 & 重壤 & 稍紧 & 多 \\
\hline & $110 \sim 170$ & 钻积层 & 7.5YR 6/4 & 重壤 & 紧实 & 少 \\
\hline & 170 202 & 母质 & 7.5YR 4/4 & 轻壤 & 紧实 & 少 \\
\hline \multirow{3}{*}{ LPM } & $0 \sim 22$ & 表层 & $10 \mathrm{YR} 5 / 4$ & 砂壤 & 疏松 & 中 \\
\hline & $22 \sim 65$ & 心土层 & 10YR 6/4 & 中壤 & 稍紧 & 中 \\
\hline & $65 \sim 120$ & 母质 & $10 \mathrm{YR} 4 / 4$ & 轻壤 & 稍紧 & 少 \\
\hline
\end{tabular}


(Thompson 和 Oldfield, 1986). HIRM, SIRM 及 S-ratio 每两或三个样品(依具体情况)测量一个样品.

磁化率高温变化曲线 $(\chi-T)$ 可以用来指示样品中 磁性矿物类型(Evans 和 Heller, 2003; Liu 等, 2005), 使用捷克 Agico 公司生产的 KLY-3S 磁化率仪和高温 控制系统测量, 精度为 $3 \times 10^{-8} \mathrm{SI}$, 测量磁场为 200 $\mathrm{Am}^{-1}$, 频率为 $875 \mathrm{~Hz}$, 测量温度从 $40 \sim 700^{\circ} \mathrm{C}$, 升温 速率约为 $12^{\circ} \mathrm{C} \mathrm{min}$, 为避免样品在加热过程中氧 化, 测量在氩气环境中进行, 氩气流量为 $50 \mathrm{~mL}$ $\min ^{-1}$.

漫反射光谱(diffuse reflectance spectra, DRS)分 析可用于计算土壤中针铁矿和赤铁矿的含量, 因此 为了更好对样品中的主要硬磁性矿物(针铁矿和赤铁 矿)进行定量化研究, 我们使用 Cary 5000 UV-VIS-IR spectrophotometer (Varian Inc., Palo Alto, CA)对样品 进行 DRS(漫反射光谱)测量, 光谱范围 300 2600 nm, 步长为 $0.5 \mathrm{~nm}$, 扫描速度为 $300 \mathrm{~nm} \mathrm{~min}{ }^{-1}$. 土壤样品 碾磨后过 200 目篎, 放入圆柱形测量器待测. 对测量
数据进行二阶求导, 分别将二阶导数曲线中 424 和 $535 \mathrm{~nm}$ 处与其后一个波峰的差值定义为 $I_{424}$ 和 $I_{535}$, 可分别用来指示样品中针铁矿和赤铁矿含量, DRS 原 理和数据处理详见 Torrent 等(2007).

\section{3 结果}

\section{1 磁学及漫反射光谱参数随土壤剖面深度变化}

剖面 LP 和 LPM 磁学参数随深度变化如图 2 所 示(图 2(a), 改自 Liu 等, 2012). $\chi, M_{\mathrm{s}}$, SIRM 和 ARM 可以反映样品中磁性矿物含量(Dekkers, 1997; Liu 等, 2007; Maher, 2007), 该组参数在 $\mathrm{A}$ 和 $\mathrm{C}$ 两层中较为 低平, 在 $\mathrm{B}$ 层曲线起伏, 呈现高值. $\chi_{\mathrm{FD}}$ 对样品中成土 作用生成的细粒 VSP(SD 和 SP 边界)磁性颗粒敏感 (Dekkers, 1997; Worm, 1998), 可用来指示样品中 SP 颗粒的含量, 其值也在剖面 B 层较高. $\chi_{\mathrm{fd}} \%$ 主要受样 品中细粒磁性矿物的含量和粒径分布影响, 这是由 于 $\chi_{\mathrm{fd}}$ 反映的是粒径范围很小的 VSP 磁性矿物含量,
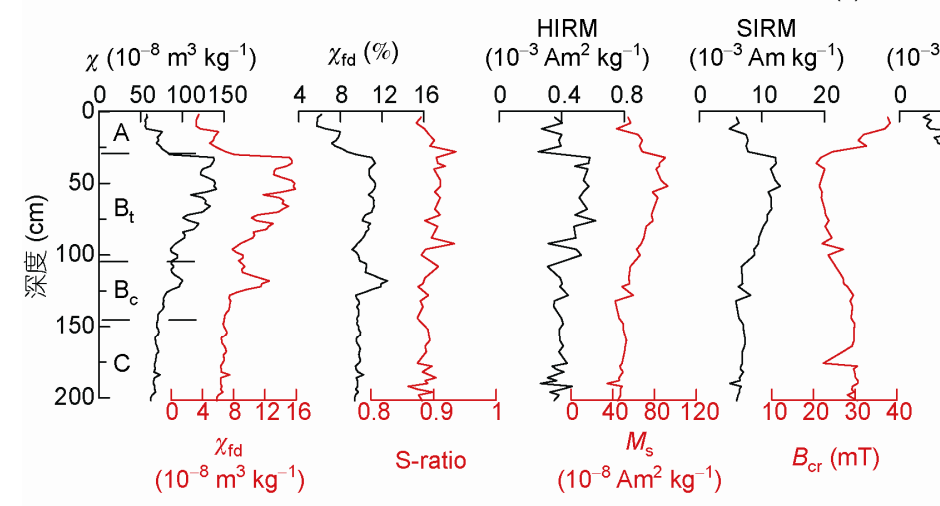

$\mathrm{ARM}$
$\mathrm{Am}^{2} \mathrm{~kg}$
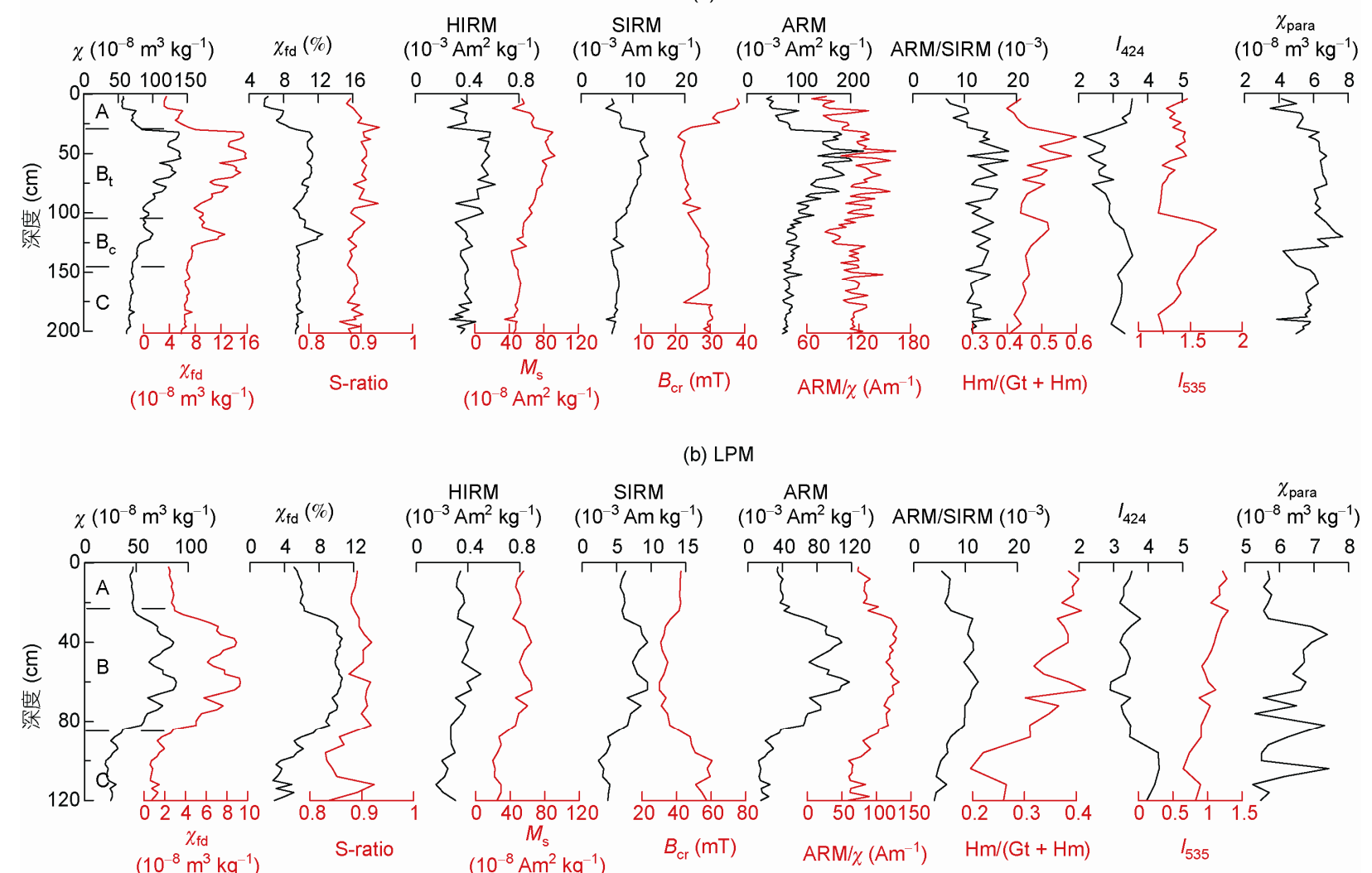

ARM
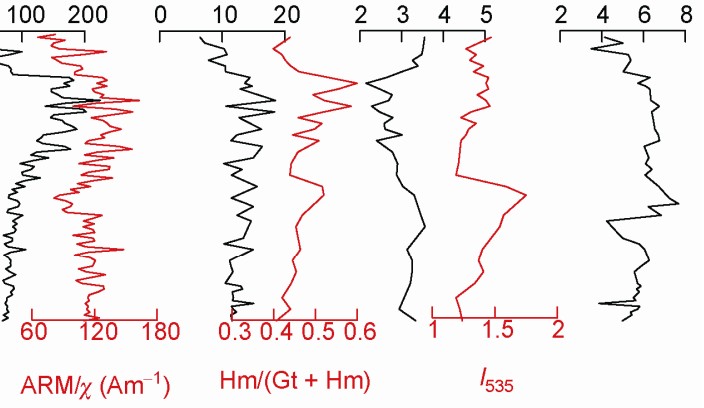

图 2 $\left(10^{-8} \mathrm{Am}^{2} \mathrm{~kg}^{-1}\right)$
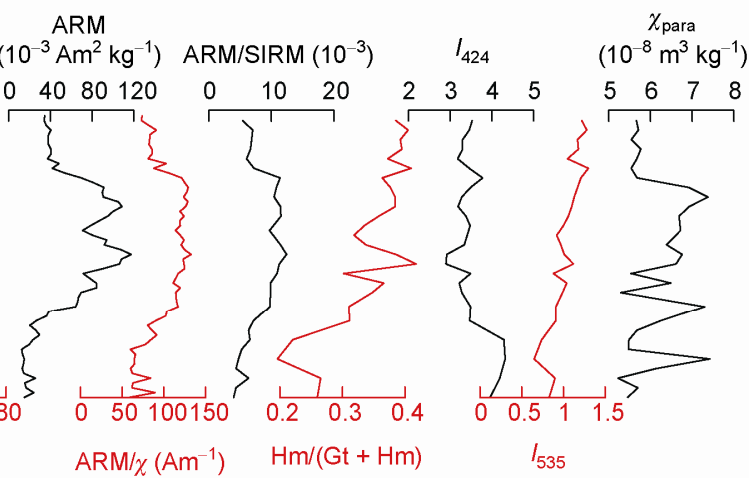

土壤剖面 LP 和 LPM 磁学及漫反射光谱参数随深度变化 
当粒径分布变大时, $\chi_{\mathrm{fd}} \%$ 值变小 (Worm, 1998; 刘青松等, 2007). LP 剖面 $\chi_{\mathrm{fd}} \%$ 变化范围为 $6 \%$ 12\%, 在 $\mathrm{A}$ 层最低, $\mathrm{B}$ 层最高, $\chi_{\mathrm{fd}} \%$ 在 $\mathrm{B}$ 和 C 层变化不明显; LPM 剖面 $\chi_{\mathrm{fd}} \%$ 变 化范围为 3\% 10\%, B 层较高, $\mathrm{A}$ 和 C 层相对较低.

S-ratio 可以反映样品中亚铁磁性矿物(如磁铁矿 和磁赤铁矿)的相对含量(Thompson 和 Oldfield, 1986), 数值分布在 0.9 左右, 说明这两个土壤剖面磁学性质 主要由亚铁磁性矿物主导. HIRM 可以反映样品中反 铁磁性矿物含量(主要为赤铁矿), 在本研究中 HIRM 随深度变化的趋势与 $\chi$ 类似. $B_{\mathrm{cr}}$ 随深度的变化趋势与 $\chi$ 相反, 在发生层 B 值较小, 这说明成土作用生成的 磁性矿物比较“软”. 顺磁性磁化率 $\chi_{\mathrm{para}}$ 表示样品中顺 磁性和反铁磁性矿物磁化率, 其变化趋势与 $\chi$ 类似.

$\mathrm{ARM} / \chi$ 可以指示样品中亚铁磁性矿物颗粒的大小 (Banerjee 等, 1981; King 等, 1982), 对于粒径大于 SD 的 颗粒, 其比值随着颗粒的减小而增大, 其高值代表样品 中以细粒稳定单畴颗粒(SSD)为主, 低值反映样品中以 粗粒的假单畴(PSD)或多畴(MD) 颗粒为主(Evans 和 Heller, 2003), 两个剖面 $\mathrm{ARM} / \chi$ 在 B 层呈现比较大. 因 为 SP 颗粒对 ARM 和 SIRM 都没有贡献, 使用 $\mathrm{ARM} / \mathrm{SIRM}$ 可以避免由于 SP 颗粒产生的不确定性(Liu 等, 2007; Maher, 2007), 通常较低的比值反映了较粗的 $\mathrm{MD}$ 颗粒. ARM/SIRM 随深度变化趋势与 $\mathrm{ARM} / \chi$ 类似, 两个剖面的 ARM/SIRM 均在 B 层呈现高值.

$I_{424}$ 和 $I_{535}$ 可分别用来指示样品中针铁矿和赤铁 矿的相对含量. 剖面 LP 和 LPM 的 $\mathrm{I}_{424}$ 随深度变化趋 势与 $\chi$ 相反. LPM 剖面 $\mathrm{I}_{535}$ 随深度增加而降低. LP 剖 面在深度 $110 \mathrm{~cm}$ 左右 $\mathrm{I}_{535}$ 呈现高值, 与此同时 $\chi, \chi_{\mathrm{fd}}$ 和 $\chi_{\mathrm{fd}} \%$ 都有高值出现, 表明有成土磁性矿物生成. 暖 干的环境有利于赤铁矿生成, 相反冷湿环境有利于 针铁矿生成(Cornell 和 Schwertmann, 2003), 因而赤 铁矿相对含量指标 $\mathrm{Hm} /(\mathrm{Hm}+\mathrm{Gt})$ 可以用来指示这两种 磁性矿物生成环境. 两个剖面 $\mathrm{Hm} /(\mathrm{Hm}+\mathrm{Gt})$ 随深度变 化与 $\chi$ 类似, 对于剖面 $\mathrm{LP}, \mathrm{Hm} /(\mathrm{Hm}+\mathrm{Gt})$ 变化范围为 $0.4 \sim 0.6$, 而 LPM 该比值的变化范围为 $0.2 \sim 0.4$, 说明 LPM 剖面中赤铁矿的相对含量较低.

\section{2 等温剩磁获得曲线(IRM)}

LP 和 LPM 剖面各发生层代表性样品 IRM 获得 曲线如图 3(a)和(b)所示. LP 各层样品 IRM 曲线形状 基本一致, 当外场为 $0.3 \mathrm{~T}$ 时获得了大约 95\%饱和磁 化强度, 这表明 LP 各层样品磁学性质由亚铁磁性矿
物(如磁铁矿和磁赤铁矿) 主导. LPM 剖面各层样品 IRM 曲线与 $\mathrm{LP}$ 类似, 均在外场为 $0.3 \mathrm{~T}$ 基本达到饱 和, C 层样品 LPM106 除外, 其在 $0.3 \mathrm{~T}$ 获得 $90 \%$ 饱和 磁化强度, 外场从 0.3 1 $\mathrm{T}$ 时剩磁逐渐升高(图 3(b)), 说明样品中矫顽力高的磁性矿物含量较其他发生层 高, 这可能是由于 LPM 剖面底层样品成土作用较弱.

\subsection{Day 图}

$M_{\mathrm{rs}} / M_{\mathrm{s}}$ 和 $B_{\mathrm{cr}} / B_{\mathrm{c}}$ 这 2 个比值参数与剩磁状态和磁各 向异性来源有关, 可以指示磁性矿物的粒度和形状 (Day 等, 1977). 理论上对于随机取向、无相互作用单轴 $\mathrm{SD}$ 磁性晶粒来说, 剩磁比值 $M_{\mathrm{rs}} / M_{\mathrm{s}}$ 值为 0.5 , 矫顽力比 值 $B_{\mathrm{cr}} / B_{\mathrm{c}}$ 都是 1.09 , 与消磁系数无关; $\mathrm{MD}$ 晶粒的 $M_{\mathrm{r} s} / M_{\mathrm{s}}$ 不到 0.05 , 矫顽力比将超过 4.0; SP 晶粒的剩磁比最低, 但矫顽力比值超过 10.0(Dunlop 和 Carter-Stiglitz, 2006). LP 和 LPM 样品均落在 Day 图的 PSD 区域(图 4), 但是 LPM 的样品比 LP 更靠近 MD 区域.
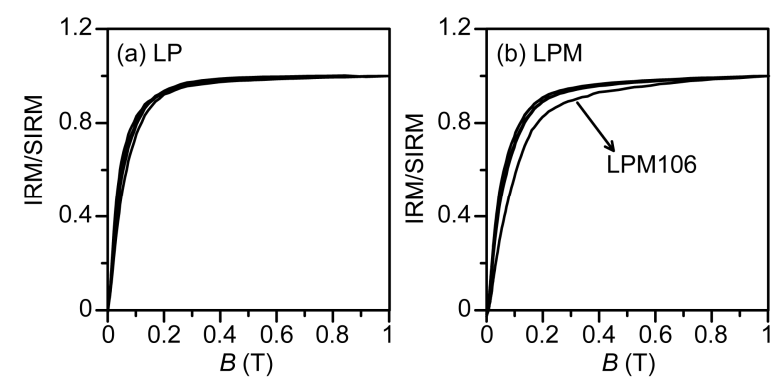

图 $3 \mathrm{LP}$ 和 LPM 各发生层代表性样品等温剩磁曲线 LPM106 表示该样品采样深度为 106 104 cm

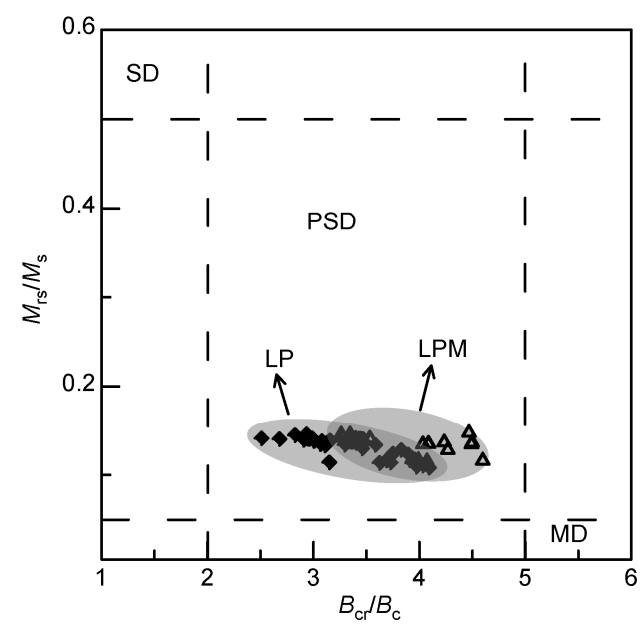

图 4 土壤剖面 LP 和 LPM 的 Day 图 实心菱形为 LP 样品, 空心三角形为 LPM 样品 


\section{4 高温 $\chi-T$ 曲线}

LP 和 LPM 剖面各层样品 $\chi-T$ 曲线如图 5 所示. 对于各层样品, 冷却曲线磁化率明显比加热曲线高, 表明土壤样品加热过程中生成了大量亚铁磁性矿物 (Deng 等, 2001; Liu 等, 2005, 2010). 除样品 LP120 外, 所有样品的加热曲线磁化率从常温到 $300^{\circ} \mathrm{C}$ 缓慢升高, 这是由于土壤样品中 SD 磁性颗粒解阻导致(Dunlop
和 Özdemir, 1997), LP120 在这一段缓慢下降可能是 由于样品中的顺磁性矿物较多, SD 颗粒相对较少. 从 $300 \sim 450^{\circ} \mathrm{C}$, 磁化率迅速下降, 这可能是因为强磁 性磁赤铁矿受热转化生成弱磁性的赤铁矿导致; 之 后随着温度的继续上升, 磁化率迅速升高, 到 $560^{\circ} \mathrm{C}$ 左右达到最高, Deng 等(2000)和 Liu 等(2005)认为这 是由于土壤中的粘土矿物在加热过程中生成磁性较 强亚铁磁性矿物； $560 ~ 580^{\circ} \mathrm{C}$ 左右，样品磁化率急剧
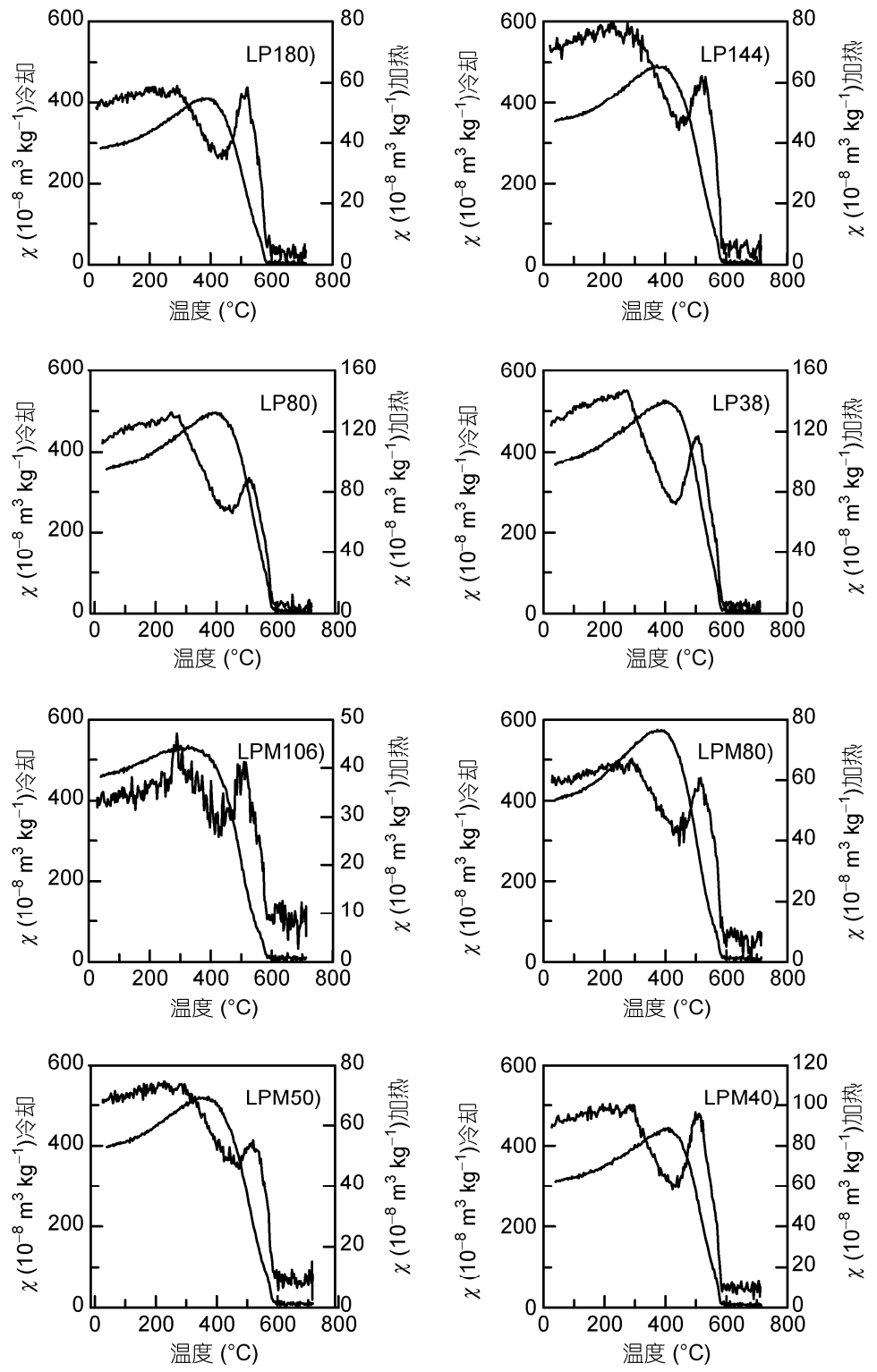
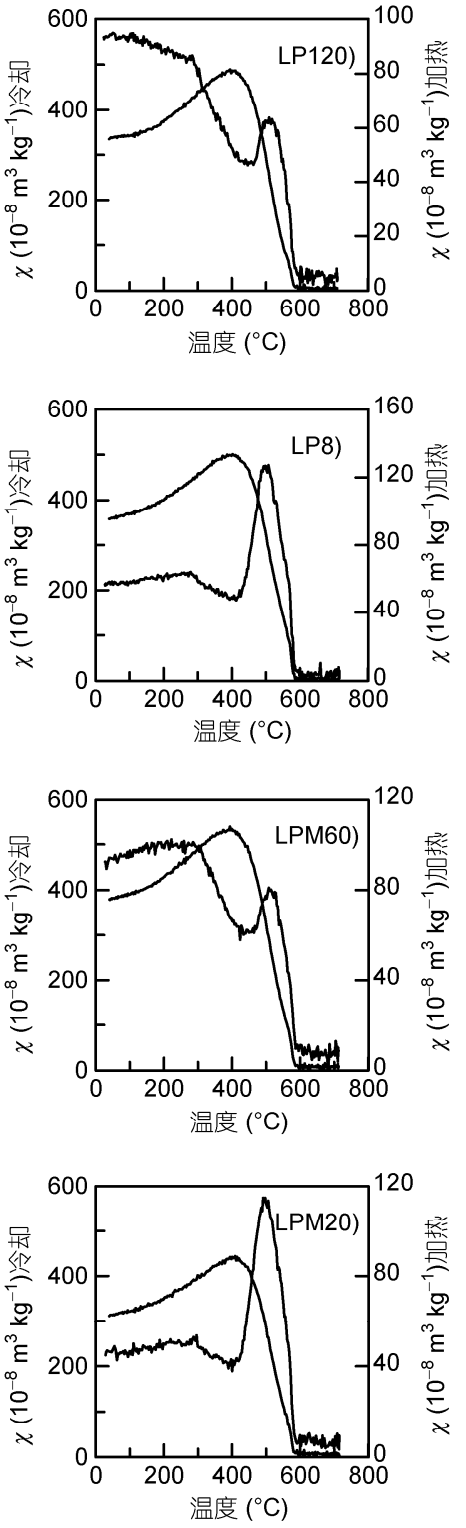

图 5 剖面 LP 和 LPM 各发生层代表性样品 $\chi-T$ 曲线

粗线代表加热曲线, 细线代表冷却曲线; 左边纵轴对应冷却曲线, 右边纵轴对应加热曲线; 样品编号代表样品的采集深度, 如 LPM106 表示 该样品采集深度为 106 104 cm 
下降到接近零, 指示了磁铁矿存在, 冷却曲线磁化率 在 $580^{\circ} \mathrm{C}$ 急剧升高也证实了这一点.

\section{5 磁性参数相关图}

剖面 LP 和 LPM 的 $\chi$ 与 $\chi_{\mathrm{FD}}$ 相关图分别如图 6(a) 和(c)所示. 剖面 LP 分为 1 104 和 106 202 cm 两部分, 两部分 $\chi$ 与 $\chi_{\mathrm{FD}}$ 线性拟合高度相关 $\left(R^{2}\right.$ 分别为 0.997 和 $0.963)$, 斜率相近分别为 0.15 和 0.157 , 在 $x$ 轴截距分 别为 $33.7 \times 10^{-8}$ 和 $26.8 \times 10^{-8} \mathrm{~m}^{3} \mathrm{~kg}^{-1}$; 剖面 LPM 表层 1 26 cm 样品(图 6(c)中阴影部分)可能受到干扰, 在 $\chi$ 与 $\chi_{\mathrm{FD}}$ 相关图中偏离整体趋势, $28 \sim 120 \mathrm{~cm}$ 样品 $\chi$ 与 $\chi_{\mathrm{FD}}$ 高度相关 $\left(R^{2}=0.997\right)$, 在 $x$ 轴截距为 $16.9 \times 10^{-8} \mathrm{~m}^{3} \mathrm{~kg}^{-1}$, 斜率为 $0.131 . \chi_{\mathrm{FD}} \%$ 反映样品中磁性矿物粒径分布 (Worm, 1998; 刘青松等, 2007, 2009), 但土壤母质中 存在 MD 颗粒磁性矿物和顺磁性粘土矿物使得 $\chi_{\mathrm{FD}} \%$ 值受到干扰, $\chi-\chi_{\mathrm{FD}}$ 相关图拟合斜率不受干扰(图 6(a) 和(c))更能反映土壤剖面成土作用生成矿物的粒径分
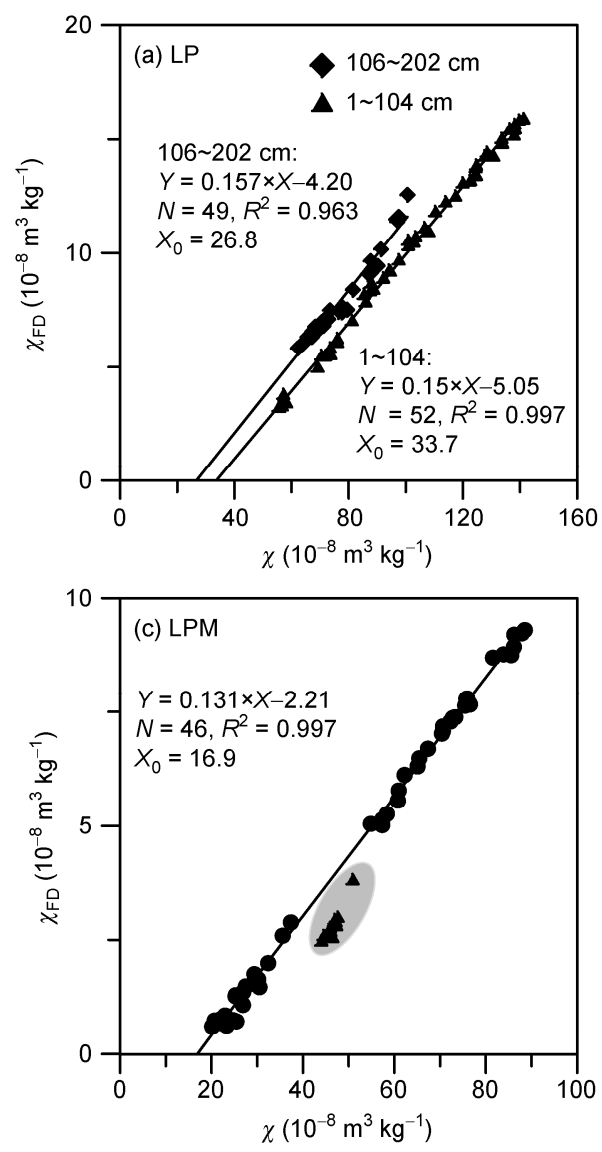

布. LP 和 LPM 拟合斜率相近, 说明坡度对成土作用 生成细粒磁性矿物粒径分布影响不大，两个剖面成 土作用生成磁性矿物的机制应该相似.

剖面 LP 和 LPM 的 $\chi_{\mathrm{FD}}-\mathrm{ARM}$ 相关图如图 6(b)和 (d)所示. 剖面 LP 的上半段 (1 104 cm) $\chi_{\mathrm{FD}}$ 和 ARM 高 度相关 $\left(R^{2}=0.90\right)$, 线性拟合直线在 $y$ 轴截距为 $10.13 \times 10^{-6} \mathrm{Am}^{2} \mathrm{~kg}^{-1}$ ( $\chi_{\mathrm{FD}}$ 为 0 ), 其下半段两个参数相 关度低 $\left(R^{2}=0.19\right)$, 可能原因为 $125 \sim 202 \mathrm{~cm}$ 处剖面成 土作用很弱, $\chi_{\mathrm{FD}}$ 随深度无明显变化; 剖面 LPM 样品 $\chi_{\mathrm{FD}}$ 和 $\mathrm{ARM}$ 高度相关 $\left(R^{2}=0.992\right)$, 在 $y$ 轴截距为 $7.53 \times 10^{-6} \mathrm{~A} \mathrm{~m}^{2} \mathrm{~kg}^{-1}$.

$\chi$ 与 ARM 相关图可以指示磁性矿物粒径, 被称 为 King-plot(Banerjee 等, 1981). ARM 对稳定 SD 颗粒 非常敏感, 而 $\chi$ 对 SP 和 PSD/MD 颗粒敏感. 因此, $\mathrm{ARM} / \chi$ 可以指示样品中亚铁磁性矿物晶粒大小, 比 值随着颗粒减小而增大，其高值代表样品中以细粒 稳定单畴颗粒(SSD)为主，低值反映样品中以粗粒的
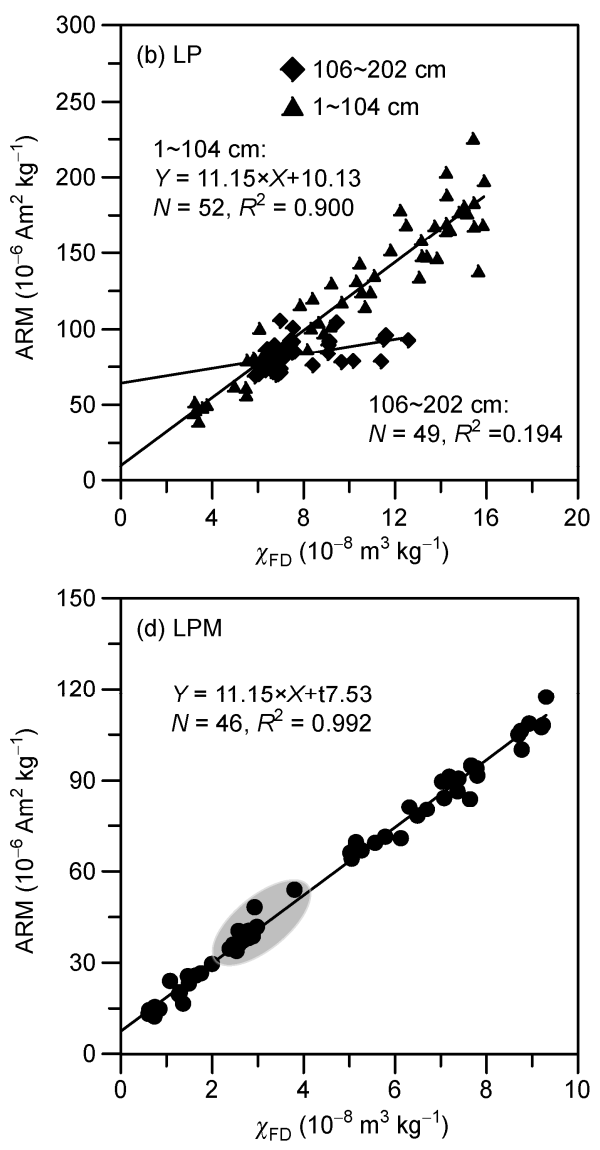

图 $6 \mathrm{LP}$ 和 LPM 剖面 $\chi-\chi_{\mathrm{FD}}$ 和 $\chi_{\mathrm{FD}}-\mathrm{ARM}$ 相关图

(a) 和 (c)中 $X_{0}$ 为对应拟合线在 $x$ 轴的截距; (c) 和(d)中阴影部分为 LPM 表层 $0 \sim 26 \mathrm{~cm}$ 样品. $P<0.05$ 
PSD 或 MD 颗粒为主(Liu 等, 2007; Maher, 2007). 两 个剖面 King-plot 分别如图 6(a)和(b)所示, 在 $x$ 轴截 距分别为 $12.41 \times 10^{-6}$ 和 $27.81 \times 10^{-6} \mathrm{~A} \mathrm{~m}^{2} \mathrm{~kg}^{-1}$ (ARM 为 0 时), 在除去剖面背景值后 $\mathrm{ARM} / \chi$ 变化均匀, 相关 性很高 $\left(R^{2}\right.$ 分别为 0.994 和 0.900), 说明 LP 和 LPM 磁 性颗粒粒径分布较窄，线性拟合 $\mathrm{ARM} / \chi$ 斜率相近分 别为 1.47 和 1.67 .

ARM/SIRM 比值对亚铁磁性矿物粒度敏感, SP 颗粒对 ARM 和 SIRM 没有贡献, 使用 ARM/SIRM 可 以避免 SP 颗粒带来的不确定性, 通常低比值反映了 粒径较粗的 MD 颗粒. LP 和 LPM 剖面 ARM-SIRM 相 关图如图 7(c)和(d)所示, 两个剖面 ARM 和 SIRM 高 度相关, 但 LP 相关性较 LPM 低 $\left(R^{2}\right.$ 分别为 0.974 和 $0.778), \mathrm{ARM} / \mathrm{SIRM}$ 线性拟合斜率分别为 20.18 和 14.73 , 在 $x$ 轴截距分别为 $3.47 \times 10^{-3}$ 和 $2.13 \times 10^{-3} \mathrm{~A} \mathrm{~m}^{2} \mathrm{~kg}^{-1}$ (ARM 为零时), 说明 LP 剖面磁性矿物粒径比 LPM 剖 面的小.
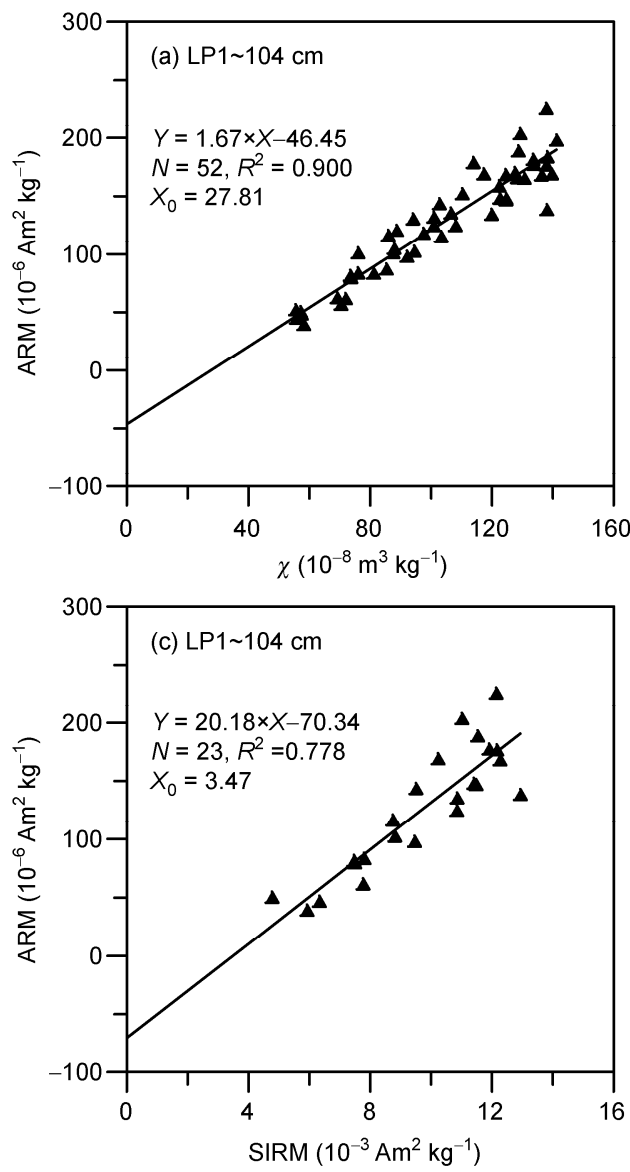

LP 与 LPM 的 $\chi_{\mathrm{FD}}-\mathrm{HIRM}$ 和 ARM-HIRM 相关图 如图 8 所示. $\chi_{\mathrm{FD}}(\mathrm{ARM})$ 和 HIRM 分别指示样品中亚铁 和反铁磁性矿物信息, 对于 $\chi_{\mathrm{FD}}-\mathrm{HIRM}$ 和 ARM-HIRM 相关图，拟合的斜率表示成土作用生成细粒亚铁磁性 矿物(磁铁矿和磁赤铁矿) 和反铁磁性矿物(赤铁矿)含量 比值. LP 和 LPM 的 $\chi_{\mathrm{FD}}-\mathrm{HIRM}$ 相关图线性拟合斜率相 似, 分别为 0.287 和 $0.289 \mathrm{Am}^{-1}, \mathrm{ARM}-\mathrm{HIRM}$ 相关图线 性拟合斜率也相似分别为 0.35 和 0.33 .

\section{4 讨论}

一般而言，土壤剖面表层磁性较底层高，这是由 于成土作用过程中生成了大量细粒亚铁磁性矿物 (SP 和 SD 颗粒)(Liu等, 2004; Liu等, 2012; Maher 和 Taylor, 1988; Zhou 等, 1990). 剖面 LP 和 LPM 表层土壤磁性 ( $\chi$, SIRM，ARM 等)均比底层高, 剖面 LP 在深度 $120 \mathrm{~cm}$ 左右 $\chi, \chi_{\mathrm{FD}}$, 和 $\chi_{\mathrm{FD}} \%$ 显示高值, 通过 $\chi-\chi_{\mathrm{FD}}$ 相关图
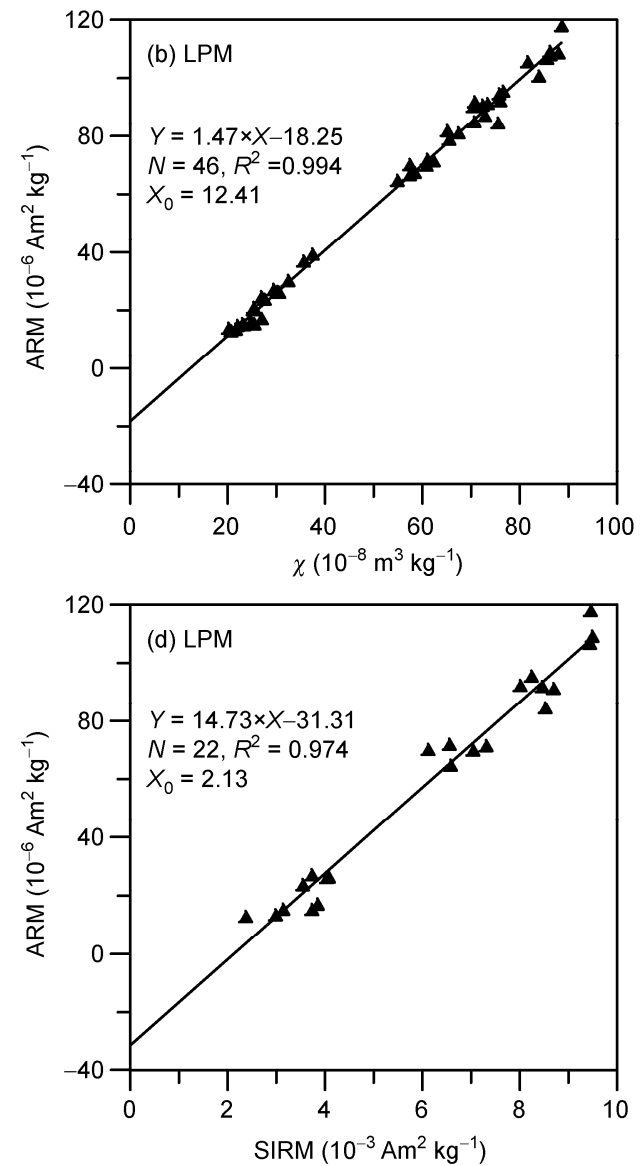

图 7 剖面 LP 上段与 LPM 的 $\chi$-ARM 和 SIRM-ARM 相关图 $P<0.05$ 

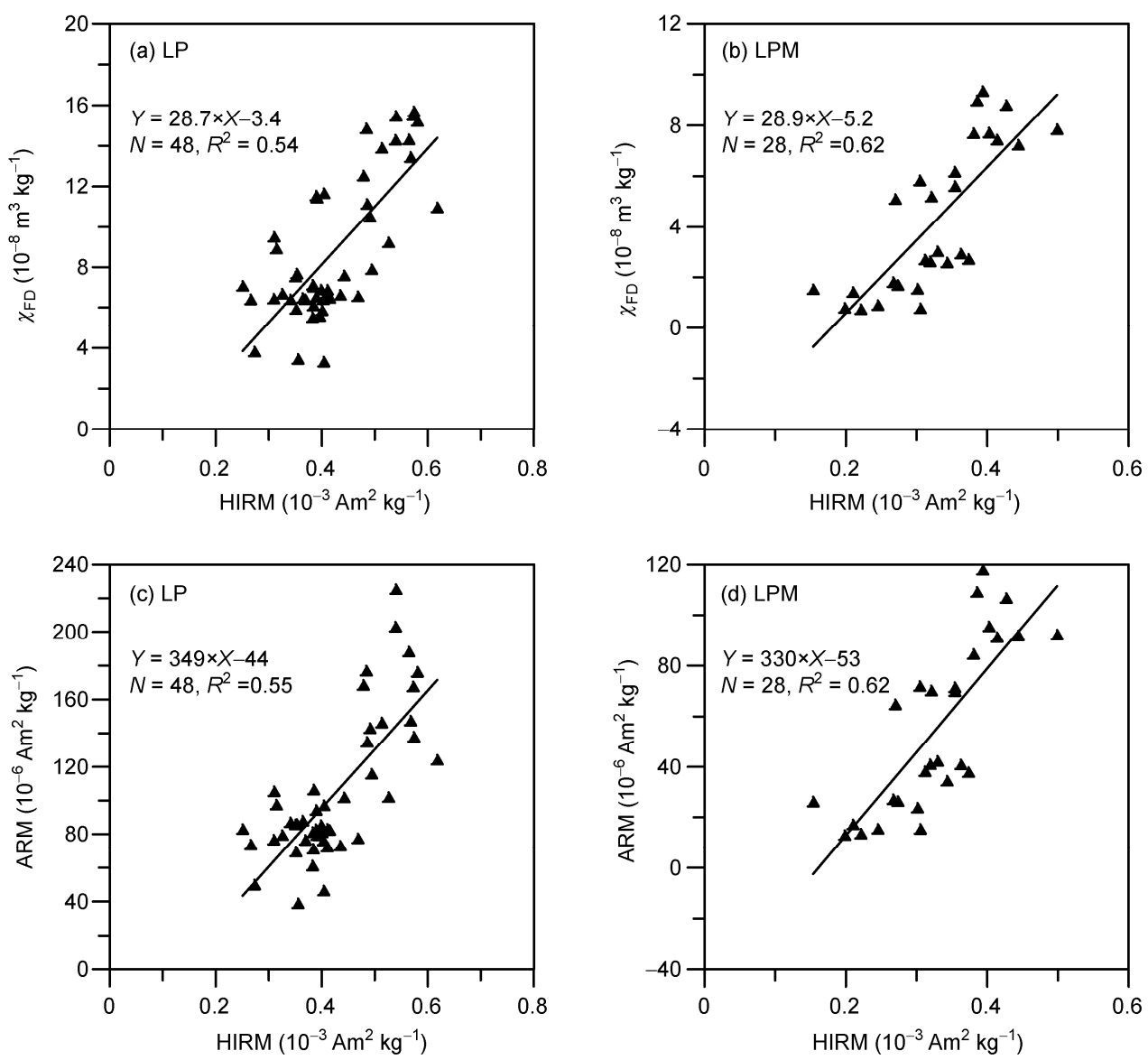

图 8 剖面 LP 与 LPM 的 $\chi_{\mathrm{FD}}-\mathrm{HIRM}$ 和 ARM-HIRM 相关图所有拟合 $P<0.05$

分析, LP 以 $104 \mathrm{~cm}$ 为界可以分为 1 104 和 106 202 cm 两段, 下半段剖面 $\mathrm{B}$ 层的成土作用较弱, 这有可能由 于该段土壤形成时成土因素(气候)发生过一次短的 改变(降雨量增加), 使得其成土作用在 $120 \mathrm{~cm}$ 稍微 增强. LPM 剖面由于坡度较大, 土壤表层受到雨水作 用影响加强, 表层 1 26 cm 受到干扰, 在 $\chi-\chi_{\mathrm{FD}}$ 相关 图上, 这部分样品位于拟合线之下 ( $\chi_{\mathrm{FD}}$ 较正常值低).

在排水良好、空气充足的土壤中, $\mathrm{Fe}^{2+}$ 离子通过 水解作用从铁的硅酸盐中析出, 然后被迅速氧化生 成水铁矿 (一种结晶度低的水合氧化物), 继而在周围 各种因素作用下, 通过不同的途径转化成磁铁矿、磁 赤铁矿、赤铁矿和针铁矿 (Cornell 和 Schwertmann, 2003), 土壤中这几种磁性矿物组成和含量受其周围 环境控制(即成土因素). 在土壤成土作用过程中对土 壤性质影响最大的因素是气候, 尤其是降雨量 (Balsam 等, 2004), 不仅决定母质的风化程度与速率,
还与其周围植被和微生物分布关系很大；母质控制 土壤中初始铁离子含量, 是土壤磁性矿物形成的基 础; 地形影响赋含在土壤中的雨水含量和保持时间, 此外还可能影响土壤侵蚀速率和母质风化速率 (Dixon 等, 2012), 从而影响土壤中磁性矿物性质; 时 间与土壤的发育程度密切相关(Fine 等, 1989).

通过建立磁学参数与降雨量之间的转换方程重 建古降雨量, 前提是土壤剖面除气候外其他成土因 素相同或者影响很小, 所以需要具体评估各种成土 因素土壤剖面磁学性质的影响. $\chi$ 是最常用来指示降 雨量的参数, Balsam 等(2011)发现在全球亚热带和温 带地区土壤的 $\chi$ 随降雨量变化趋势为先升高后降低. 在前人的研究中, 一个地点往往只选择一个土壤样 品重建古降雨量, 而 $\chi$ 随土壤剖面深度不同变化很大 (图 2), 这可能会加大研究的不确定性. 在同一地点 的两个土壤剖面, 磁化率也可能差别很大. 在本研究 
区域中, 由于土壤剖面坡度差异, 导致 LP 和 LPM 两 个土壤剖面虽然相距很近, 降雨量和温度等气候条 件相同, 但是磁性差异也很大, 剖面 LP 的 $\chi$ 最大值 和平均值均约为 LPM 两倍. 因此在使用 $\chi$ 作为指示 古降雨量指标时需要慎重, Geiss 等(2008)提出在使用 磁性参数增强相对值建立气候转换方程指示古降雨 量, 可能比指示磁性矿物含量的磁性参数更为合理, 他使用 ARM/SIRM 指示降雨量, 获得了比较好的结 果. 但在本研究中两个剖面 LP 和 LPM 的 ARM/SIRM 比值相差较大, 这可能是因为 ARM/SIRM 包含粒径 信息，且受母质中大颗粒磁性矿物影响.

剖面 LP 指示磁性矿物含量参数 $\left(\chi, M_{\mathrm{s}}\right.$, SIRM 和 $\mathrm{ARM}$ )的平均值与最大值均明显比 LPM 高(图 2), 如 LP 的 $\chi$ 平均值或者最大值均约为 LPM 的两倍. 两个 土壤剖面时间、植被和气候等三个成上因素相同, 根 据 Jenny(1941)土壤发生理论, 影响其性质的成土因 素为母质和地形. LPM 剖面水平坡度较 LP 大, 雨水 在 LPM 赋存时间较短, 使得其粘土矿物中铁离子析 出量减少, 从而在其他成土因素相同的条件下, 生成 的磁性矿物较 LP 少. 在 $\chi-\chi_{\mathrm{FD}}$ 相关图中, 线性拟合直 线在 $x$ 轴截距可以作为土壤剖面 $\chi$ 背景值(Liu 等, 2004), LP 背景值大约为 LPM 的两倍(图 6(a)和(c), LP 和 LPM 在 $x$ 轴截距分别为 $33.7 \times 10^{-8}$ 和 $16.9 \times 10^{-8} \mathrm{~m}^{3}$ $\left.\mathrm{kg}^{-1}\right)$, 指示磁性矿物含量参数 $\left(\chi, M_{\mathrm{s}}, \mathrm{SIRM}\right.$ 和 ARM) 与土壤剖面形成时背景值关系密切. 在重建古降雨 量时, 需要与土壤背景值无关, 只受降雨量影响的磁 学参数. 土壤剖面因成土作用导致的磁性增强与背 景值比值受背景值影响较小(Geiss 等, 2008), 定义土 壤剖面磁化率增强参数 $E_{\chi}=\left(\chi_{\text {mean }}-\chi_{\text {background }}\right) / \chi_{\text {background }}$, LP 和 LPM 磁化率增强比值分别为 $E_{\chi-\mathrm{LP}}=(102.4-$ 33.7) $/ 33.7=2.04, E_{\chi \text {-LPM }}=(55.3-16.9) / 16.9=2.27$. 成土 作用过程不仅生成 SP 颗粒的磁性矿物, 也会生成 SD 的, 通过 ARM-SIRM 相关图(图 7(c)和(d)) 得到 SIRM 背景值, 并计算出 SIRM 增强比值, $E_{\text {SIRM-LP }}=$
$(9.7-3.47) / 3.47=1.80, \quad E_{\text {SIRM-LPM }}=(6.1-2.13) / 2.13=1.86$. 本研究中 LP 和 LPM 两个剖面 $\chi$ 和 SIRM 磁性增强比 值 $E_{\chi}$ 和 $E_{\mathrm{SIRM}}$ 相似, 受土壤母质和坡度影响很小, 相 比磁性参数(如 $\chi, \mathrm{ARM}$ ) 可能更好反映降雨量对土壤 磁学性质影响.

土壤剖面成土作用磁铁矿与赤铁矿含量比值 $(\mathrm{Mg} / \mathrm{Hm})$ 受土壤背景较小, 而且包含了成土过程生成 的亚铁和反铁磁铁矿物信息, 也可以用来指示古降 雨量(Balsam 等, 2004; Liu 等, 2007; Torrent 等, 2006). 在本研究中, 分别用 $\chi_{\mathrm{FD}}$ 和 $\mathrm{ARM}$ 指示成土作用生成 $\mathrm{SP}$ 和 SD 亚铁磁性矿物 (磁铁矿和磁赤铁矿), 用 HIRM 指示反铁磁性矿物 (赤铁矿). LP 和 LPM 的 $\chi_{\mathrm{FD}} /$ HIRM 拟合斜率分别为 $28.7 \times 10^{-5}$ 和 $28.9 \times 10^{-5} \mathrm{~A} \mathrm{~m}^{-1}$, $\mathrm{ARM} / \mathrm{HIRM}$ 的拟合斜率分别为 0.35 和 0.33 (图 8). 结 果验证了 Torrent 等(2006)和 Liu 等(2007)提出的 $\mathrm{Mg} / \mathrm{Hm}$ 比值受控于降雨量的观点. $\chi_{\mathrm{FD}} / \mathrm{HIRM}$ 和 $\mathrm{ARM} / \mathrm{HIRM}$ 拟合斜率受背景值及斜率影响较小, 表 明使用土壤剖面成土作用生成的磁铁矿与赤铁矿含 量比值 $(\mathrm{Mg} / \mathrm{Hm})$ 作为降雨量的指示参数有很大潜力.

\section{5 结论}

本文通过对陕西延安同一地点两个坡度不同的 土壤剖面进行详细的磁学和漫反射光谱研究, 探讨土 壤剖面坡度对其磁学性质的影响及其在古降雨量重建 中的应用, 结果发现: (1) 土壤剖面的坡度影响整个土 壤剖面磁性矿物含量, 但成土磁性矿物类型和粒径分 布相似, 不受土壤剖面坡度影响; (2) 传统指示古降雨 量的参数 $\chi$, 指示土壤中磁性矿物含量, 误差较大, 在 使用时需谨慎; (3) $\chi$ 和 SIRM 因成土作用增加的倍数 $E_{\chi}$ 和 $E_{\mathrm{SIRM}}$, 可以反映降雨量对土壤磁学性质的影响; (4) 相关图 $\chi_{\mathrm{FD}}-\mathrm{HIRM}$ 和 ARM-HIRM 的拟合斜率 $\chi_{\mathrm{FD}} / \mathrm{HIRM}$ 和 $\mathrm{ARM} / \mathrm{HIRM}$ 包含成土作用生成的亚铁和 反铁磁性矿物信息, 在指示降雨量上有很大潜力.

致谢感谢靳春胜博士在采样时及姜兆霞同学在论文完成时提出宝贵建议, 感谢四位审稿人的宝贵意见.

\section{参考文献}

郭兆员. 1992. 陕西土壤, 北京: 科学出版社. 520

刘秀铭, 刘东生, 夏敦胜, 等. 2007. 中国与西伯利亚黄土磁化率古气候记录一氧化和还原条件下的两种成土模式分析. 中国科学 D 辑: 地球科学, 37: 1382-1391

刘青松, 邓成龙. 2009. 磁化率及其环境意义. 地球物理学报, 52: 1041-1048

2046 
刘青松, 邓成龙, 潘永信. 2007. 磁铁矿和磁赤铁矿磁化率的温度和频率特性及其环境磁学意义. 第四纪研究, 27: 955-962

Balsam W, Ji J, Chen J. 2004. Climatic interpretation of the Luochuan and Lingtai loess sections, China, based on changing iron oxide mineralogy and magnetic susceptibility. Earth Planet Sci Lett, 223: 335-348

Balsam W L, Ellwood B B, Ji J, et al. 2011. Magnetic susceptibility as a proxy for rainfall: Worldwide data from tropical and temperate climate. Quat Sci Rev, 30: 2732-2744

Banerjee S K, King J W, Marvin J A. 1981. A rapid method for magnetic granulometry with applications to environmental studies. Geophys Res Lett, 8: $333-336$

Blundell A, Dearing J A, Boyle J F, et al. 2009. Controlling factors for the spatial variability of soil magnetic susceptibility across England and Wales. Earth-Sci Rev, 95: 158-188

Chesworth W. 2008. Encyclopedia of Soil Science. Netherlands: Kluwer Academic Pub

Cornell R M, Schwertmann U. 2003. The Iron Oxides: Structure, Properties, Reactions, Occurrences and Uses. Weinheim: Wiley-VCH

Day R, Fuller M, Schmidt V A. 1977. Hysteresis properties of titanomagnetites: Grain-size and compositional dependence. Phys Earth Planet Inter, 13: 260-266

Dearing J A, Hay K L, Baban S M J, et al. 1996. Magnetic susceptibility of soil: An evaluation of conflicting theories using a national data set. Geophys J Int, 127: 728-734

Dekkers M J. 1997. Environmental magnetism: An introduction. Geol Mijnbouw, 76: 163-182

Deng C, Zhu R, Jackson M, et al. 2001. Variability of the temperature-dependent susceptibility of the Holocene eolian deposits in the Chinese Loess Plateau: A pedogenesis indicator. Phys Chem Earth (A), 26: 873-878

Deng C L, Zhu R X, Verosub K L, et al. 2000. Paleoclimatic significance of the temperature-dependent susceptibility of Holocene loess along a NW-SE transect in the Chinese Loess Plateau. Geophys Res Lett, 27: 3715-3718

Dixon J L, Hartshorn A S, Heimsath A M, et al. 2012. Chemical weathering response to tectonic forcing: A soils perspective from the San Gabriel Mountains, California. Earth Planet Sci Lett, 323-324: 40-49

Dunlop D J, Özdemir Ö. 1997. Rock Magnetism: Fundamentals and Frontiers. Cambridge: Cambridge University Press. 573

Dunlop D J, Carter-Stiglitz B. 2006. Day plots of mixtures of superparamagnetic, single-domain, pseudosingle-domain, and multidomain magnetites. J Geophys Res, 111: B12S09, doi: 10.1029/2006jb004499

Evans M E, Heller F. 2003. Environmental Magnetism: Principles and Applications of Environmagnetics. Amsterdam: Academic Press

Fine P, Singer M J, La Ven R, et al. 1989. Role of pedogenesis in distribution of magnetic susceptibility in two California chronosequences. Geoderma, 44: 287-306

Geiss C E, Egli R, Zanner C W. 2008. Direct estimates of pedogenic magnetite as a tool to reconstruct past climates from buried soils. J Geophys Res, 113: B11102, doi: 10.1029/2008JB005669

Han J M, Lu H Y, Wu N Q, et al. 1996. The magnetic susceptibility of modern soils in China and its use for paleoclimate reconstruction. Stud Geophys Geod, 40: 262-275

Heller F, Liu T S. 1982. Magnetostratigraphical dating of loess deposits in China. Nature, 300: 431-433

Heller F, Liu T S. 1984. Magnetism of Chinese loess deposits. Geophys J Int, 77: 125-141

Heller F, Liu T S. 1986. Palaeoclimatic and sedimentary history from magnetic susceptibility of loess in China. Geophys Res Lett, 13: 1169-1172

Jenny H. 1941. Factors of Soil Formation: A System of Quantitative Pedology. New York: McGraw-Hill. 281

King J W, Banerjee S K, Marvin J A, et al. 1982. A comparison of different magnetic methods for determining the relative grain size of magnetite in natural materials: Some results from lake sediments. Earth Planet Sci Lett, 59: 404-419

Kukla G, Heller F, Liu X M, et al. 1988. Pleistocene climates in China dated by magnetic susceptibility. Geology, 16: 811-814

Liu Q, Jackson M J, Yu Y, et al. 2004. Grain size distribution of pedogenic magnetic particles in Chinese loess/paleosols. Geophys Res Lett, 31 : L22603, doi: 10.1029/2004GL021090

Liu Q, Deng C, Torrent J, et al. 2007. Review of recent developments in mineral magnetism of the Chinese loess. Quat Sci Rev, 26: 368-385

Liu Q, Deng C, Yu Y, et al. 2005. Temperature dependence of magnetic susceptibility in an argon environment: Implications for pedogenesis of Chinese loess/palaeosols. Geophys J Int, 161: 102-112

Liu Q, Roberts A P, Larrasoaña J C, et al. 2012. Environmental magnetism: Principles and applications. Rev Geophys, 50: RG4002, doi: 10.1029/2012RG000393

Liu Q, Torrent J, Morrás H, et al. 2010. Superparamagnetism of two modern soils from the northeastern Pampean region, Argentina and its paleoclimatic indications. Geophys J Int, 183: 695-705

Liu Z F, Liu Q S, Torrent J, et al. 2012. Testing the magnetic proxy $\chi_{\mathrm{FD}} / \mathrm{HIRM}$ for quantifying paleorainfall in modern soil profiles from Shaanxi 
Province, China. Global Planet Change, http://dx.doi.org/10.1016/j.gloplacha.2013.04.013

Maher B A. 2007. Environmental magnetism and climate change. Contemp Phys, 48: 247-274

Maher B A, Taylor R M. 1988. Formation of ultrafine-grained magnetite in soils. Nature, 336: 368-371

Maher B A, Thompson R, Zhou L P. 1994. Spatial and temporal reconstructions of changes in the Asian palaeomonsoon: A new mineral magnetic approach. Earth Planet Sci Lett, 125: 461-471

Maher B A, Alekseev A, Alekseeva T. 2002. Variation of soil magnetism across the Russian steppe: Its significance for use of soil magnetism as a palaeorainfall proxy. Quat Sci Rev, 21: 1571-1576

Thompson R, Oldfield F. 1986. Environmental Magnetism, London: Allen and Unwin. 227

Torrent J, Barrón V, Liu Q. 2006. Magnetic enhancement is linked to and precedes hematite formation in aerobic soil. Geophys Res Lett, 33: L02401, dio: 10.1029/2005g1024818

Torrent J, Liu Q, Bloemendal J, et al. 2007. Magnetic enhancement and iron oxides in the upper luochuan loess-paleosol sequence, Chinese Loess Plateau. Soil Sci Soc Am J, 71: 1570-1578

Worm H U. 1998. On the superparamagnetic-stable single domain transition for magnetite, and frequency dependence of susceptibility. Geophys J Int, 133: 201-206

Zhou L P, Oldfield F, Wintle A G, et al. 1990. Partly pedogenic origin of magnetic variations in Chinese loess. Nature, 346: 737-739 